\title{
Midwives' views and experiences of providing healthy eating advice to pregnant women: a qualitative content analysis of semi-structured interviews
}

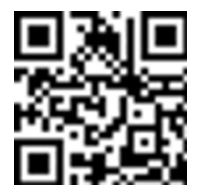

Original article

Shwikar Mahmoud Etman Othman ${ }^{\mathrm{a}}$, Julie-Anne Fleet ${ }^{\mathrm{b}}$, Mary Steen $^{\mathrm{b}}$, Rasika Jayasekarab

aObstetrics and Gynaecology Nursing Department, Faculty of Nursing, South Valley University Egypt, Qena 83523, Egypt

${ }^{b}$ UniSA Clinical \& Health Sciences, University of South Australia, Adelaide, South Australia 5001, Australia

Received: 10 March 2020; Accepted: 22 May 2020; Published: 20 December 2020

\begin{abstract}
Objective: To explore midwives' views on how they provide healthy eating education to pregnant women after attending a healthy eating education workshop/webinar.

Methods: A qualitative descriptive approach was utilized. Semi-structured interviews were conducted to explore the views and experiences of midwives on providing healthy eating education for pregnant women. A purposive sample of six midwives was interviewed face-to-face, and one was conducted by telephone interview. Data were analyzed through qualitative conventional content analysis. Results: Midwives described their views and experiences of factors that impacted their role in providing healthy eating education. They identified three categories: perceived role of midwives, health literacy, and model of care.

Conclusions: Knowledge and confidence of midwives improved after attending the workshop/webinar on healthy eating education. Findings suggested that midwives perceived their role as important in providing nutrition education. However, time and resources were highlighted as challenges when providing healthy eating education for pregnant women. The availability of health literacy and model of care were significant factors in enabling midwives to adequately provide this education. Midwives acknowledged a need for further education in areas of vegan diet, cultural food preferences for ethnic minority groups, and regular updates on national healthy eating guidelines.
\end{abstract}

Keywords: midwives $\bullet$ healthy eating guidelines $\bullet$ nutrition policy $\bullet$ qualitative $\bullet$ content analysis $\bullet$ education $\bullet$ maternity health services

(c) Shanxi Medical Periodical Press.

How to cite this article: Othman SME, Fleet JA, Steen M, Jayasekara R. Midwives' views and experiences of providing healthy eating advice to pregnant women: a qualitative content analysis of semi-structured interviews. Front Nurs. 2020;4:345-358. 


\section{Introduction}

Adequate nutrition during preconception and prenatal periods are essential for healthy pregnancy outcomes. ${ }^{1,2}$ A well-balanced diet and physical activity are important to maintain a healthy pregnancy. ${ }^{2}$ However, many pregnant women do not receive accurate information and sufficient healthy eating education during prenatal appointments. ${ }^{3}$

Midwives are perceived to be at a unique position to provide healthy eating education and advice to mothers and families. ${ }^{4,5}$ The International Confederation of Midwives stipulate that the midwives' role should include assessment of the nutritional status of pregnant women and their health behaviors. ${ }^{6}$ This assessment should include providing advice and education about dietary intake and nutritional supplements, such as folic acid, which is based on the most recent evidence-based guidelines. ${ }^{6}$ Interactions between a midwife and pregnant woman take place during antenatal appointments and also classes in which midwives provide advice and support to meet a woman's individual needs. ${ }^{7}$ However, midwives have challenges to overcome, such as limitedtime assigned for appointments and discussing sensitive issues, for example obesity, which may impede their role in providing healthy eating education. ${ }^{8}$ Therefore, midwives have previously suggested that more support, guidance, and assistance are required to provide updated healthy eating education and advice to pregnant mothers. ${ }^{9}$

It is a requirement that midwives participate in continual professional education (CPD). ${ }^{4}$ However, it has been reported that only a small number of midwives have had opportunities to engage in CPD for nutritional education, while others utilized the internet as an alternate resource for information. ${ }^{8}$ Interestingly, only a few studies have explored the views of midwives and their perceived role in providing healthy eating education and advice to pregnant women. ${ }^{8}$ One study in Australia that explored self-reported data relating to healthy eating education found that approximately $80 \%$ of midwives received some undergraduate nutrition education. However, the education was described as limited and lacked focus on topics such as nutrition assessment and nutrition for vulnerable groups of pregnant women such as those with medical conditions or vegans, vegetarians or food preferences for different ethnic minorities. $^{8}$

No previous research has been conducted with midwives to explore their views and experiences across several time-points after attending a healthy eating education program. Therefore, a sequential exploratory mixed methods design ${ }^{10}$ was utilized, which included two phases. Phase 1 was a pre-, immediate, and post-test intervention study that used questionnaires to assess the level of knowledge and confidence of midwives before and after the development and provision of a healthy eating education workshop/ webinar. This education program was conducted to enhance the knowledge and confidence of midwives in providing nutrition education about healthy eating during pregnancy. The education program was provided either at a workshop or at a webinar. Midwives who were working and residing in South Australia were invited to participate, and findings demonstrated that their level of knowledge and confidence increased after attending the education program and was maintained at 6-8 weeks. The findings from Phase 1 were reported in a separate paper. ${ }^{11}$ Phase 2 was a qualitative descriptive study that aimed to obtain an in-depth insight into midwives' views after attending a $2 \mathrm{~h}$ healthy eating education workshop/ webinar. This second phase also aimed to explore the midwives' experiences on how they provided healthy eating education to pregnant women after attending the education program. This article reports on Phase 2.

\section{Methods}

A qualitative descriptive design was selected to provide a clear description of the phenomena (midwives' views and experiences $)^{12}$ and uses an iterative process of data collection and analysis of participants responses to semi-structured questions. ${ }^{13}$

A qualitative descriptive design was undertaken to explore the views and experiences of midwives in providing healthy eating education to pregnant women through semi-structured interviews. The interview schedule was guided by a systematic review ${ }^{14}$ and phase one of the mixed-methods study. Questions were open-ended, focusing on midwives' experiences and their role in providing healthy eating education to pregnant women, including education for women who are vegan or vegetarian or have cultural food preferences. Additional questions were asked about using standard guidelines and resources and potential barriers that may impede midwives from providing healthy eating education. Midwives were also invited to give feedback on the content, mode, and structure of the healthy eating education workshop/webinar.

\subsection{Participants and setting}

Midwives who attended a healthy eating workshop/ webinar were invited to participate in a follow-up semistructured interview (6-8 weeks post-education). From a total of 19 midwives who completed the healthy eating education workshop/webinar and post-follow-up questionnaire, a purposive sample of seven midwives 
consented and were interviewed. Of these, six midwives were interviewed face-to-face, and one preferred a telephone interview. The selection criteria included midwives who were working in metropolitan and rural antenatal clinics, midwifery group practices, and/or undertook prenatal parent education classes in South Australia and had completed the healthy eating workshop/webinar.

The interviews were audio-recorded and ranged from 25 to $45 \mathrm{~min}$. Face-to-face and telephone interviews were conducted by the primary researcher, in a private room at the University of South Australia (UniSA), and Women and Children's Hospital to maintain privacy and confidentiality of participants.

The interviews were transcribed verbatim. Data was collected in December 2018, and the transcription and analysis were completed between April 2019 and August 2019 , by the primary researcher and two co-authors. Data saturation was achieved when no new information/ codes were generated which guided the decision to stop recruiting new participant midwives.

\subsection{Data analysis}

All interviews were transcribed by the primary researcher. Two authors reviewed transcripts for accuracy. Data were analyzed using conventional content analysis ${ }^{15}$ to explore midwives' knowledge, attitudes, and behaviors related to healthy eating education and health benefits. ${ }^{16}$ Content analysis described in this article aimed to achieve a clear broad description of midwives' views and experiences after attending the healthy eating education, with results being presented in categories. This approach was utilized through a threephase framework for representing the content analysis process ${ }^{17}$ as follows:

\subsubsection{Preparation phase}

An inductive approach, including open coding, creating main categories, abstractions to describe aspects of the whole content, was used. The primary researcher read and re-read transcripts to become familiar with the entire data set. ${ }^{15}$ Data were coded, and categories and abstractions were identified. ${ }^{15,17}$ Inter-rater reliability was confirmed by two authors carrying out data analysis to reduce bias during the preparation and organization phases. The final categories produced were agreed upon by all researchers.

\subsubsection{Organizing phase}

Codes were sorted into main categories and subcategories using NVivo 12 plus software.

\subsubsection{Reporting the analysis process and the results}

A final report was written to provide definite, logical, classified conclusions of the story data tells within and across codes and categories.

\subsection{Demonstrating rigor (trustworthiness) in qualitative data}

Five strategies and principles were used to ensure and maintain rigor and trustworthiness, these include credibility, dependability, confirmability, transferability, and reflexivity. ${ }^{18,19}$ Several strategies were used to ensure the credibility of the data, such as prolonged engagement, triangulation, and member checking..$^{20}$ On prolonged engagement, the first author (SO) provided the healthy eating education and conducted the interview with the midwives to build trust and familiarity with the researcher. The first author completed the initial data analysis and data triangulation and two co-researchers carried out data coding and interpretation. Member checking was conducted during the interview to seek clarification, and examples were provided to confirm the correct meaning.

Transferability was maintained through a sufficient sample size, clear explanation of data collection, and analysis as per the developed and published protocol. ${ }^{10}$ A detailed description of the participants' characteristics, experiences, and views is provided with quotes and recommendations for future research. An audit trail for dependability and confirmability was maintained through all steps, and procedures are described in the published study protocol. ${ }^{10}$

Reflexivity included critical self-reflection to maintain a balance between researcher and research relationship. The authors are registered midwives and nurses who are engaged in midwifery and nursing education, teaching and clinical practice, they are engaged in conducting quantitative, qualitative and mixed-methods research and publish in reputable journals with up to 35 years of experience.

\subsubsection{Reliability for content analysis}

The reliability of the content analysis was conducted through three steps: stability, reproducibility, and accuracy. ${ }^{21,22}$ Stability was achieved as results were checked several times to ensure accuracy. Reproducibility was maintained, where co-author (JF) conducted independent data analysis to replicate findings. The primary researcher and co-authors conducted cross-checking of the transcripts with the audiotapes to ensure the accuracy of the records. 


\section{Results}

\subsection{Participants characteristics}

Nineteen midwives were invited to participate in a semi-structured interview after attending an education workshop/webinar, from those seven midwives provided written consent and took part in an interview. All midwives were female and had completed a workshop/ webinar and post-education questionnaire. Participants' details are presented in Table 1.

\subsection{Midwives' views categories}

Three principal categories were identified from the content analysis. The first category was midwives' perceived role including midwives' knowledge and confidence, education delivery, and available resources. The second category was limited health literacy including midwives' level of education, need for CPD, and the pregnant

\begin{tabular}{llc}
\hline MW & Place of practice & $\begin{array}{c}\text { Years of } \\
\text { experience }\end{array}$ \\
\hline \hline MW1 & Tertiary Hospital (Nursery/Postnatal) & 8 \\
MW2 & Tertiary Hospital (Midwifery Group Practice) & 13 \\
MW3 & Tertiary Hospital (Antenatal clinic) & 24 \\
MW4 & Tertiary Hospital (Antenatal clinic/Antenatal & 27 \\
& Educator) & \\
MW5 & Tertiary Hospital (Antenatal clinic) & 21 \\
MW6 & Midwifery Educator/Midwifery private practice & 14 \\
MW7 & Tertiary Hospital (Midwifery Group Practice/ & 35 \\
& Birth Centre/Midwifery private practice) & \\
\hline
\end{tabular}

Table 1. Characteristics of midwives who participated in the interview. women perceived level of knowledge and education. The third category was models of care which midwives described impacted their ability to develop relationships, allocate time and follow-up, particularly if they did not work in a continuity of care model. See Figure 1.

\subsubsection{Midwives' role in providing healthy eating education}

The opening interview question asked midwives about their perceived role of providing healthy eating education to pregnant women. Midwives were asked to describe how they usually provided healthy eating education and what resources were available to them.

\subsubsection{Midwives'knowledge and confidence to provide healthy eating education}

All midwives perceived to provide healthy eating education to pregnant women as a vital role. Midwives described their relationship with pregnant women, acknowledging that they often were the main trusted source of information, and they felt they influenced their behavior.

"... As very important! As midwives are often the main source of this sort of information...I would give dietary advice daily ... in my role." MW3

"I think ... in a bit of position of power being a midwife ... I think ... certainly, have influence [on healthy eating]..." MW7

Although providing healthy eating education to pregnant women was considered as part of the midwives'

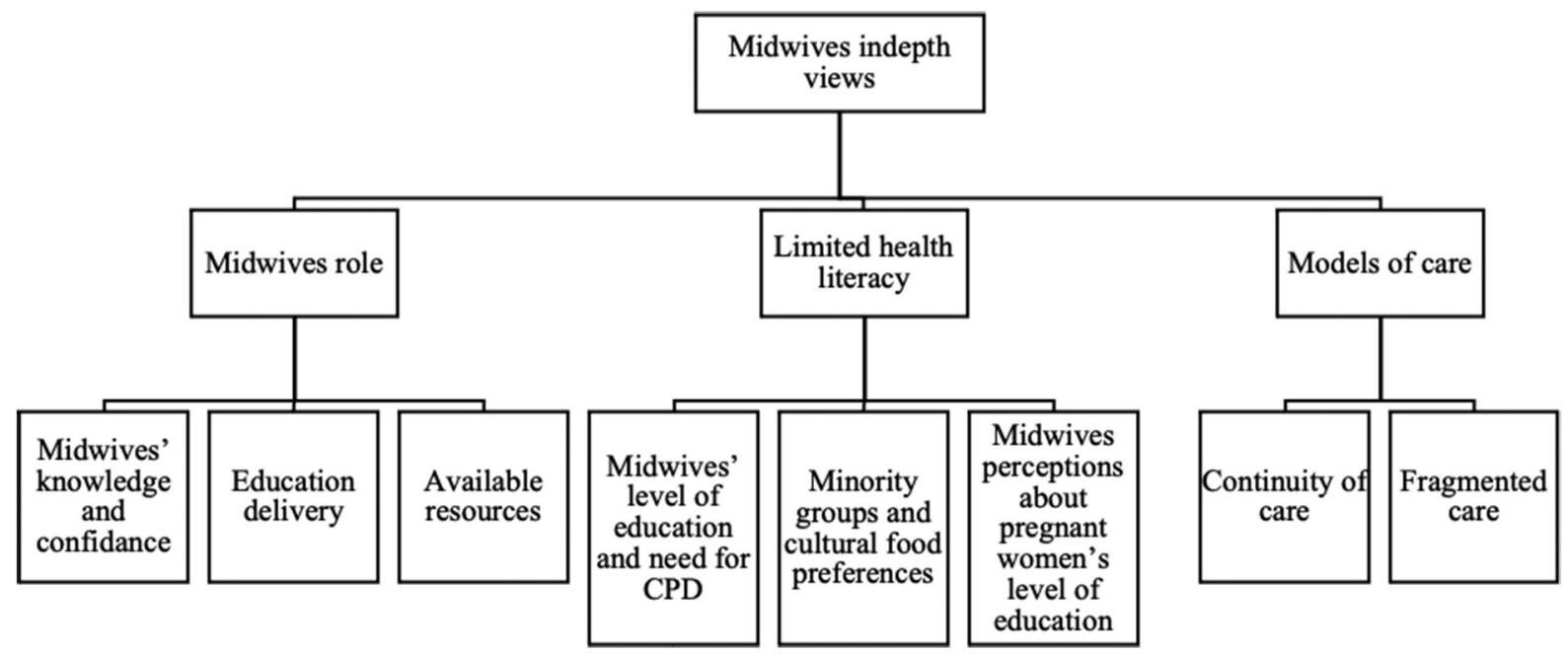

Figure 1. Midwives views after attending healthy eating education workshops/webinar. 
role, midwives discussed barriers to provide this education sufficiently. For example, how motivated or unmotivated women are to acquire knowledge and understand lifestyle choices.

"(my role is) very hit and miss ... I think my influence on them is ... depending on the situation and how motivated they are ... but it's also a little bit of insight, often I think people just do not have the insight into knowing what lifestyle choices they've take?..." MW5

Some midwives felt that healthy eating education was not undertaken effectively, and not all midwives felt it was their role to influence women's food choices.

“... I'm not going to tell you [pregnant woman] what to do, because I don't think that's my role either, as they are adults having babies..." MW4

“... I think we're dealing with a lot of ... midwives' ignorance as well ... So, it is left to the midwife ... we really only just scratching the surface with [dietary] information .... "... it's labour intensive healthy eating a lot of the time and there're a lot of people [midwives] who are not interested in putting a lot of effort into it." MW5

All midwives reported an increase in confidence and were more knowledgeable after attending the education and more aware of providing healthy eating education to pregnant women; in particular, those who are vegans and vegetarians would use updated resources provided.

"I think I just need to be more conscious about maybe bringing it [nutrition advice] into my antenatal appointments ... so again it's just ... having the confidence to talk to them about diet ... and I certainly feel a lot more confident in certain respects ... as I said ... vegans, and vegetarians and things to talk to them a bit more now with confidence." MW2

\subsubsection{Education delivery}

All midwives reported confidence to provide general nutrition education, but not specific information for atrisk populations such as vegans, vegetarians, or women with gestational diabetes. Most referred them to receive education from a dietitian or diabetic educator. Midwives perceived their role to then monitor women and followup at each antenatal appointment.

“... I keep my discussion relatively general and the most specific is when l'm advising women who have the potential or diagnosed pregnancy diabetes ..." MW3
"I've got very basic knowledge about nutrients levels but when it comes to other complexes, I would not hesitate to go okay; this person really should go and see a dietitian, for example, an overweight [woman]." MW4

"I often refer them [vegans/vegetarians] on to a dietitian because I would not feel comfortable in knowing how to advise them on their balanced diet with their increased pregnancy needs ..." MW5

When midwives discussed their level of education to provide this general information, most depended on their undergraduate education, available resources at their place of employment and or their personal experiences.

“... I think I just did it from my personal knowledge, I mean I'm probably going back to my midwifery education and maybe I hadn't even checked whether things had moved on which obviously they had, but I suppose again because I don't get involved in the initial booking where it's very important to sort of educating women on what they should not eat ..." MW2

"I'm also a type 2 diabetic so ... I'm quite comfortable also talking to diabetic women ... I don't have any problem talking to vegans or vegetarians. I was raised vegetarian, I have been vegan for 18 months ..." MW3

Consistent with the midwives' role of providing healthy eating education, three midwives offered a general education about food safety, macronutrients, portion size, what to eat and what to avoid, and different types of supplements.

“... tailoring the education to what women need ... you've got your healthy eating ... exercise ... sleep ... rest ..."MW1

"... we would give them information sheets on good sources of iron for people who are vegetarian ... there is some detailed information about iron, about listeria, about um mercury in fish, all those precautionary things around that. So, we've got information that we give. So, we were always talked with women one to one about that ..." MW4

\subsubsection{Available resources}

When midwives provide healthy eating education, they tend to use available resources in the hospital such as 
brochures, pamphlets, information sheets, or browsing websites.

“... websites ... most people have access to internet these days ... so yeah, I guess just brochures, pamphlets and an online sort of things" MW1

“... we do have a very quick talk about it [healthy eating] at triage, we tell them about the pamphlet and the information that it has in it ... unless people sort of stop you and say look l've got some questions ..." MW5

Most midwives reported using resources as an addition to education they provided to pregnant women for example, brochures were provided and discussed to ensure that women understood the content.

"... I discuss with her first and then leave her with the brochures and give her a copy ..., so that make sure that she understands ..."MW1

However, most midwives reported that available information provided through the hospital was descriptive but not comprehensive enough for all pregnant women.

“... I don't think that the dietary information was as extensive in the written information when we would talk to them. But in the actual written information, I don't know that we are giving them really extensive advice in written information, so I don't think we're giving them enough." MW6

While midwives interviewed used available nutrition information resources within the hospital, only two midwives referred to using national or international healthy eating guidelines when discussing nutrition with pregnant women.

"... probably refer to the perinatal practice guidelines ... I've got a few government brochures ... that's talking ... more about listeria and food safety ..." MW4

"In the past, if I didn't have information I normally Google it while I was in a clinic ... we have the Better Health Channel ... so I probably just do what, do some googling ..."MW6

\subsubsection{Limited health literacy}

Midwives identified some limitations in their nutrition knowledge, recognizing the need for CPD, and referral to the guidelines and education about cultural food choices. In addition, midwives reported difficulties in educating pregnant women from diverse backgrounds and their level of education. At times, midwives made assumptions about pregnant woman's health literacy.

\subsubsection{Midwives level of education and the need for $C P D$}

Midwives' primary role in health promotion is to provide education about food sources for pregnant women. However, most midwives explained the importance of supplements such as, vitamins and potential side effects instead of providing more detailed education about food sources.

"... I think taking the supplements is kind of the easy way
out ... because it does take time, It does take money to
plan appropriately what you're going to eat and a lot of
women are busy ... some women aren't compliant with
taking iron supplements as recommended ... because of
the side effects of that you know it causes constipation
and things like that." MW1

"... I would mention the need for iodine at every booking visit and confirm that the women are taking an appropriate supplement." MW3

An example provided by a midwife demonstrated ways to engage women about cultural food preferences and food choices, but it was found to be difficult within their role.

"l'd love to explore with women ... those in pregnancy or birth or, early after birth ... the traditions that are important and I can usually see a good scientific reason ...to agree with it ... and to encourage it, and so part of that discussion often is ... what did grandma say was important food ... I enjoy that ... I tend to find a lot of the cultural beliefs as very protective of the mother and or the mother or the baby ..." MW3

Some midwives reported low levels of knowledge and confidence before attending the workshop/webinar because they were not aware of the healthy eating guidelines or did not trust the validity of their content.

"... our knowledge is limited ... there is a lot of detail that we actually do need to be on top of ... we've done a little bit of it [education] now in service ... " " ... l've got very basic knowledge ..." MW4

"Yeah, I probably don't specifically refer to [guidelines]. I've been around long enough to see these things change in my lifetime ... So, some of the guidelines I'd take with a small pinch of salt." MW3 
“... I ... didn't even know that there were actual guidelines, l've never looked at those ..." MW7

Midwives reported the need for CPD on healthy eating education to update their knowledge and confidence about cultural food preferences, women with specific dietary requirements, and current guidelines.

"I really struggle with people with the specific dietary requirements like the vegans or the vegetarians and ... I often refer them on to a dietitian because I would not feel comfortable in knowing how to advise them on their balanced diet with their increased pregnancy needs ..." MW5

"... as midwives who are working with women antenatally that we do all [need to] stay a little bit more up to date with the information that we're giving especially when we're looking at having lots and lots of different women in our care that are not always eating traditional Western diet ... So yeah it was helpful." MW6

\subsubsection{Minority groups and cultural food preferences}

Part of the midwives' role is to provide healthy eating education to women from different backgrounds. However, a few midwives reported that they avoided offering healthy eating education to pregnant women from diverse cultural backgrounds, reporting it was not their place to influence changes to their way of life.

"Yeah I would probably try and offer it [Education]. We have a saying that's very Australian ... teaching people how to suck eggs is like teaching them stuff they already know it's like you're being condescending ... I would try and avoid that. I would not want to be telling someone their business, they know what they're doing. It's their life. It's their culture. It's their way of doing things and I don't want to be preaching or telling them anything that's not actually my ..." MW4

"... we are certainly very multicultural area and have a lot of ethnic women ... I don't tend to have too much interaction with them about what they're eating at home unless there's a problem." MW7

All midwives who provided healthy eating education to pregnant women from an ethnic minority group reported barriers such as, language, traditions, and limited understanding of cultural food preferences that impacted how to approach the education.

"I think culture [knowledge] ... might be lacking a little bit still so more resources on that, and ... particularly not just in their language but in their foods as well ... " " ... language barriers ... tradition ... what they're traditionally used to eating or taking and just a change. Change is a barrier in itself getting people to change their views, their opinions on things ..." MW1

"... it was more around their cultural norms rather than me understanding all of their different things, ... I did not understand their particular values ... it was more them teaching me." MW5

All midwives interviewed identified a need for education about cultural food preferences for pregnant women from ethnic minorities and vegans because of their perceived limited knowledge in those specific areas.

"I guess probably with women from different cultures so that would probably be the big thing ... there's a little bit of a downfall with women from different cultures and women that are a vegan as well." MW6

\subsubsection{Midwives' perceptions about pregnant women's level of education}

Regarding health literacy for pregnant women, midwives reported several assumptions about pregnant women's level of knowledge and their lifestyle behaviors. Some midwives reported that pregnant women in their model of care were well educated and motivated to seek education and advice.

“... The women are knowledgeable ... they're not stupid.

I really don't like making people feel like they're not very clever ..." MW4

“... I think for the women ... they usually very well informed women and they would usually do a lot of reading and seeking a lot of information." MW6

Nevertheless, some midwives described a few pregnant women that they were aggressive and not receptive to their education and felt they could not influence their behavior.

"Well they don't always wish to hear what l'm saying, ... I have had a very upset woman that being told that she had gestational diabetes and she was very aggressive about getting some dietary advice ..." MW3

“... there are women who don't take advice or are not interested in changing their diet ... so that affects their gestational diabetes ..." MW7

In terms of pregnant women's lifestyle and behavior, midwives described that some pregnant women used 
vitamin supplements due to their busy lifestyle, health behaviors, and cultural background.

"I think people think that they have ticked all the boxes if they take a multivitamin, I do think it's an easier option for a lot of people and I think they genuinely believe that they're doing the right thing by taking them" "... I think ... most of the gestational diabetes, some vegetarian women who have suffered the effects of anaemia are feeling better on supplements ..." MW5

"... they'll often need a supplement though. And they often come with supplements; it seems like in their culture, they'll often turn up with this bag of vitamins, that they've bought from home ..." MW4

Furthermore, some midwives assumed that pregnant women from different cultures would often eat healthier than Australian women do.

“... I think most women of other ethnicities eat much more healthy than Australian women do ... they tend to eat more fruits and vegetables ... they don't eat any processed foods, I don't know, It's just an assumption I have about ethnic groups, actually that they eat more healthy ..."MW4

\subsubsection{Models of care}

Differences in health care services influenced how education was delivered at different levels and ways of interaction with the pregnant women, midwives experienced different models of care within the hospital, and they interacted with pregnant women accordingly. These models of care discussed included continuity of care (a known midwife), and fragmented care (seeing a different clinician for antenatal appointments, birth, and postnatal care).

\subsubsection{Continuity of care}

Midwives reported that the midwifery continuity of care model was the best model of care to enable the time to educate, build a rapport, and provide follow-up care. Pregnant women who did not receive this model of care were reported as missing out.

"I think if you've not got any continuity, you're going to miss that level of care." MW4

“... so we still decline ... about 25 women a month because we haven't got the space [midwifery group practice] ..." MW2
“... if l've had them on continuity of care, I would follow them up throughout the pregnancy ... I could spend an hour with them. So, I didn't have anybody saying you can't spend more than that time with her. l've been able to spend lots of time with the women and give them really good ... education, but in public hospitals, we don't have enough time." MW6

\subsubsection{Fragmented care}

From the midwives' point of view, pregnant women who were in a fragmented care model often missed out on the benefits of the continuity of care, and more time was wasted to looking back for the previous information provided by another clinician.

“... I don't get a lot of opportunities to work with a pregnant woman and sort of provide advice to them so, because yeah I did not work in a clinic ..." MW1

“... I'm thinking you don't see that same person again, again having to go over that previous ground in your next visit and you've run out of time ..." MW4

All midwives identified barriers within the health services, which hinder them from being able to provide comprehensive education to pregnant women. These barriers included time, follow-up, and the availability of the continuity of care model.

"Continuity and time so not having the opportunity to see you again ... our visits are limited ... we have ... 1.5 hours for the first visit and probably 20 minutes for their subsequent visits. That's not really a lot of time to have a conversation to get to know somebody, to measure their tummy, to do their blood pressure like that's a limiting factor especially when you're doublebooked or you use an interpreter, or you've got 10 people on your list." MW4

"Follow up is ... more difficult, l'm not in a continuity of care model ... so, women, I see at a triage or booking visit I won't necessarily see again in the pregnancy." MW3

"... I don't get involved in the initial booking where it's very important to sort of educating women ..." MW2

This main category highlights that midwives reported their role was to provide general basic healthy eating education to pregnant women using hospital resources. This was influenced by the midwives' level of education and background. Furthermore, assumptions about limited health literacy influenced the education provided. 
Midwives reported that pregnant women's motivation or lack of motivation impacted on their relationship and ability to influence healthy eating behavior changes. In addition, midwives reported challenges within the maternity services relating to different models of care which impeded their ability to provide healthy eating education to pregnant women.

\section{Discussion}

This current study provides in-depth insights into midwives' views and experiences of providing healthy eating education to pregnant women. Findings provide an exploration of some midwives' views and barriers encountered which may impact their role when providing healthy eating education to pregnant women. Three main categories were identified: midwives' perceived role, limited health literacy, and models of care.

\subsection{Midwives' perceived role}

Midwives perceived themselves in a position to provide and influence healthy eating education as part of their daily practice at antenatal clinics; however, this perceived role is not aligned with their current level of knowledge and confidence. Midwives interviewed reported that they had general basic nutritional knowledge but identified limitations that challenged them when providing healthy eating education to pregnant women. These limitations align with previous studies findings that midwives have general nutrition knowledge. ${ }^{23,24}$ However, a literature review identified few exploratory and descriptive studies and reported that midwives lacked basic knowledge of nutrition requirements during pregnancy. ${ }^{25}$

Some midwives reported that should women need detailed nutrition advice, they would be referred to a dietician, i.e. being overweight or special dietary requirements, such a vegans and vegetarians. Reasons for referral included midwives who lacked the confidence to provide nutrition education and limited advanced nutritional knowledge. These findings align with previous studies that midwives provide general nutrition advice and refer women with specific conditions to a dietitian. ${ }^{9,23}$ However, a few midwives provided detailed nutritional education to address pregnant women's individual circumstances. ${ }^{23}$ Of the midwives who made a referral to a specialist, the most common reasons were diabetes and/or obesity, complex medical conditions, and special diets such as vegans or vegetarians pregnant women. ${ }^{23}$ All midwives provided similar examples of circumstances when they would refer to a dietician, advice given for these risk factors were often based on personal experiences rather than actively seeking professional development for these risk factors. However, all these circumstances were mentioned by midwives equally in this study.

When the midwives provided examples of educating pregnant women, all midwives discussed educating pregnant women with gestational diabetes; however, these pregnant women were also more often referred to receive healthy eating education from dietitians or diabetic educators. Interestingly, midwives did not appear to focus their education on women without medical conditions, often appearing to assume they were already educated or could access their own information. This finding is consistent with a previous study which reported that $(n=182,59.5 \%)$ of midwives would only discuss healthy eating education for women with a medical condition such as gestational diabetes. ${ }^{23}$

The current findings showed that the scope of the midwives' role was not only to provide nutrition education but also included discussion of different nutritionrelated aspects during pregnancy such as food safety, portion size, supplements, and side effects, as well as exercises sleep and rest. Furthermore, all midwives provided this education quickly at antenatal appointments, which was different from previous studies in which only some midwives were active in providing individualized healthy eating education to pregnant women ${ }^{8,9,26}$ and focused on nutrition-related aspects such as macronutrients and portion size, and exercise. ${ }^{26}$ However, it has been reported that some midwives avoid discussing nutrition-related issues such as listeria and folic acid, and junk food. ${ }^{9}$

Nevertheless, midwives' perceived role and their influential position to provide education to pregnant women may be impeded by other factors such as the women's level of motivation, health literacy, and sociodemographic characteristics. There has been a previous pre-, post-test intervention study to assess pregnant women's nutritional knowledge using nutrition education modules developed by the Ministry of Health in Turkey. ${ }^{27}$ This study revealed that according to the level of education at pre-test, the high school graduates, pregnant women had higher scores than the primary school graduates, as well as those who were illiterate and literate. The study concluded that pregnant women needed adequate and appropriate healthy eating education according to their sociodemographic characteristics. A randomized controlled trial in Iran assessed the effects of two antenatal classes (2 h sessions, 1 week apart) based on the Health Belief Model to influence the dietary behaviors of pregnant women. ${ }^{28}$ The findings of this study also reported that women's motivation is 
essential to make behavior modifications and produce excellent nutritional performance. In addition, the combination of classes and group consultations was shown to increase pregnant women's nutritional knowledge and eating behaviors.

Midwives, in the current study provided healthy eating education depending on available brochures in the hospital, their background education, and personal experience. Arrish et al. reported that from a sample size of $n=329$, midwives seek nutrition information sources from (other health care professionals ( $n=249,81.4 \%$ ), general knowledge $(n=231,75.5 \%)$, and midwifery education $(n=192,62.7 \%) .{ }^{23}$ Similarly, another study with a sample size of $n=370$ reported that midwives seek information from nutrition organizations $(n=316$, $85.6 \%)$, general knowledge $(n=307,83.2 \%)$ and midwifery education ( $n=268,72.6 \%)$ or midwifery journals $(n=236,64.0 \%) .{ }^{29}$

In terms of using available educational resources reported in the current study most midwives educated pregnant women through discussion using brochures, pamphlets, information sheets, and online resources and provided them with a written copy. This finding is similar to a previous qualitative study where pregnant women received leaflets and booklets on relevant health topics from midwives during antenatal appointments to supplement their verbal education. ${ }^{30}$ Conversely, some midwives used a passive approach as they relied on the written information, especially for pregnant women they perceived to be healthy ${ }^{9,31}$ or midwives referred to a package of brochures as part of the pregnancy booklet that contained other materials, and they did not mention or discuss the nutrition brochure. ${ }^{32}$

In the current study midwives reported they only occasionally referred to national standard guidelines for healthy eating during pregnancy or other prenatal practice guidelines as educational resources. This finding has concurred with earlier studies, which indicate that midwives did not adhere to national standard guidelines. ${ }^{23,33}$ Nevertheless, one New Zealand study identified that midwives were well-educated to discuss nutrition issues related to pregnancy and strictly used the national standard guidelines when providing healthy eating education to pregnant women. ${ }^{29}$ Whereas, some of the midwives reported that the hospital resources were descriptive, not comprehensive, and out-of-date, and they felt conflicted while using them. A previous cross-sectional postal survey study reported that hospital resources were utilized by the majority of pregnant women ( $n=246 / 350,70 \%$ ) as information sources provided by midwives to meet their needs. ${ }^{34}$ It is, therefore, important that guidelines and resources are kept up to date to enable women to access reliable information.
This main category has highlighted that midwives' reported their level of knowledge and confidence increased significantly after attending a healthy eating education workshops/webinar, in particular, to provide education to vegans and vegetarians pregnant women. This is important as previous studies suggest midwives' level of knowledge and confidence are moderate to low, in particular reinforcing the need for healthy eating education to support vegans and vegetarians pregnant women. ${ }^{23,29}$ This finding demonstrates the need to include these women and specific information in education sessions. All findings indicate that most midwives have a good understanding of the importance of healthy eating education and well-being during pregnancy. However, some midwives might lack support and training based on the most recent guidelines.

\subsection{Limited health literacy}

Health literacy is related to a person's ability to obtain, interpret, understand, and use health information to promote and maintain health status. ${ }^{35}$ Nutrition literacy is a subordinate concept of health literacy. ${ }^{36}$ In this article, limited health literacy, for example, skills required to understand and communicate health information ${ }^{37}$ was found for both midwives and pregnant women. Many factors were found to affect their role such as some midwives reported limited knowledge about nutrition guidelines, or awareness of their scope of practice, or they may have incorrect perceptions about the pregnant women's level of education. These factors may impede midwives from seeking regular CPD of recent nutrition guidelines.

Midwives' role in health promotion ${ }^{38}$, is to provide a holistic approach that includes healthy eating education and advice and to discuss nutrients from available food sources according to the pregnant woman's health needs. However, most midwives regularly provided education and recommended vitamin supplements and discussed side effects; this type of discussion is medically directed and does not provide a holistic focus for nutritional education. However, a previous study highlighted that some midwives viewed food sources and diet as the first medicine, and they recommended food sources over supplements when discussing nutrition issues with pregnant women. ${ }^{9}$

In this study midwives reported that pregnant women who used vitamin supplementations were influenced to do so by their health behavior and cultural background. Even when women are aware of pregnancy vitamin supplements, education should be provided on dietary intake and lifestyle choices. A recent Australian study 
that explored women's attitudes regarding preconception health behaviors and information preferences revealed that women perceived adopting lifestyle behaviors included a healthy diet, physical activity, and vitamin supplementations as good practice. ${ }^{39}$ Interestingly, findings from this current study suggest that midwives were making several assumptions that pregnant women were well-educated, motivated, and doing their own search for information. Midwives would then only provide education when pregnant women asked questions or when problems arise. Previous studies clarified that pregnant women were concerned that midwives made incorrect assumptions about the extent of their knowledge..$^{9,23,30}$ Therefore, pregnant women sought their own information as they felt the information provided was inaccurate or inadequate or caused confusion..$^{40-42}$

Some midwives assumed that pregnant women from different cultural backgrounds were eating healthier than Australian women. However, these assumptions were not based on scientific evidence. Previous studies found that migrant pregnant women from low or middle-income countries were often associated with the adoption of less healthy dietary habits. ${ }^{41,42}$

Further, this current study highlighted that most midwives require education for recent standard healthy eating guidelines and cultural food preferences for ethnic minority groups. Midwives identified barriers when providing education to pregnant women from an ethnic minority group, including language, traditions, change, and culture. Moreover, some midwives were not aware of other cultures' food types, and food groups, reporting they needed additional resources and education to explore different cultures. These findings confirmed the previous results from phase $1^{11}$ of this research project. Literature suggests that midwives rarely discussed healthy eating education for different cultural food groups ( $n=54 / 329,20.7 \%) .{ }^{8}$

In the United Kingdom, studies that explored midwives' perception and knowledge of maternal obesity did not view healthy eating education as a priority, midwives did not recognize the national guidelines sources, and they recognized their limitations. ${ }^{43,44}$ Further, midwives did not tailor advice for women's cultural influences, and specific diet such as vegans, vegetarians. ${ }^{43,44} \mathrm{~A}$ previous study explored the experiences of women of different ethnic backgrounds in Norway when receiving nutrition-related information. ${ }^{40}$ The study found the nutrition information provided by midwives was different to the pregnant women's original food culture and resulted in confusion; therefore, this led to women seeking their own nutrition information by themselves. ${ }^{40}$ The study concluded that nutrition communication skills should be tailored toward women's cultural background, level of nutritional knowledge and nutrition literacy. ${ }^{40}$

\subsection{Models of care}

It has been reported that the provision of health care services provided for maternity care is varied according to locations in Australia. ${ }^{45}$ These health care services are delivered at different levels, according to the workforce, the size of the maternity units, and the pregnant woman's needs. ${ }^{45}$ Midwives, in the current study described midwifery continuity of care to be the best model of care for pregnant women and her developing baby and felt women might miss valuable care when they did not have that model of care. Previous studies are consistent with the findings of this study that is reported that in the continuity of care model, midwives had the opportunity and time to discuss healthy eating education with pregnant women, and midwives were able to work on their knowledge, skills and obtain resources required for pregnant women needs. ${ }^{46,47}$ Another study revealed that the continuity of care model had several benefits for pregnant women's health outcomes, as well as benefits for midwives such as high job satisfaction and less occupational burnout. ${ }^{48}$ Cochrane and systematic reviews have concluded that pregnant women who were in the continuity of care models were less likely to experience interventions and be satisfied with their care with less adverse outcomes for them and their developing baby's health status. ${ }^{49,50}$ Moreover, pregnant women experienced building trust and empowered relationships with their midwives. ${ }^{49,50}$

According to the current findings, midwives reported that the limited availability of continuity of care models resulted in fragmented care which created barriers for them to provide healthy eating education during the booking visits and follow-up for continuous support for pregnant women. Similarly, a previous study identified few women had accessed to the continuity of care model, and it offered inequality in the public sector. ${ }^{51}$ Congruently, Arrish et al. described that midwives who worked in the public or private sector in fragmented care had many barriers that hindered them from providing effective healthy eating education and advice, such as limited time, less opportunity to see the pregnant women again or late involvement with the pregnant women. ${ }^{9}$

Findings that have emerged from the content analysis of the midwives' interviews have identified several professional and health services barriers such as nutritional knowledge, health literacy, time, and models of care, which impede them to fulfill their role in providing healthy eating education to pregnant women. 


\subsection{Strength and limitations}

The current study is the first to report on midwives' views and experience after attending a healthy eating education workshops/webinar. The interviews were conducted after 6-8 weeks to follow-up some midwives who participated in the healthy eating education to explore their views and experience when providing healthy eating education to pregnant women. Independent analysis from two authors (SO), (JF) helped to maintain and ensure rigor, as well as reduce subjectivity bias. However, the participants included only experienced midwives who had between 8 to 35 years of midwifery practice experiences, and no recent midwifery graduates were interviewed. Therefore, the findings reported in this study should be interpreted with caution to other cohorts. Further research to explore the experiences of newly graduated midwives would be beneficial.

\section{Conclusions}

Based on findings discussed in this article, midwives' level of knowledge and confidence improved after attending a healthy eating education workshop/webinar. Midwives expressed motivation to implement changes to their practice that included using updated resources and modified how they provided education to women. Midwives perceived their role to provide nutrition education as important. However, health literacy and model of care were a significant factor to adequately provide this education. Midwives acknowledged a need for further education for special dietary requirements, such as vegan and cultural preferences for ethnic minority groups and regular updates on national healthy eating guidelines. In addition, time and resources were highlighted as limiting factors to provide healthy eating education for pregnant women.

\section{References}

1. Royal College of Obstetricians and Gynaecologists. Nutrition in pregnancy updated SEP 2010, Royal College of Obstetricians and Gynaecologists; 2010. https://www.rcog.org.uk/en/guidelines-researchservices/guidelines/sip18/. Accessed July 19, 2019.

2. Widen E, Siega-Riz AM. Prenatal nutrition: a practical guide for assessment and counseling. J Midwifery Womens' Health. 2010;55:540-549.

3. Bookari K, Yeatman H, Williamson M. Informing nutrition care in the antenatal period: pregnant women's experiences and need for support. Biomed Res Int. 2017;2017:4856527.

\section{Recommendations}

Providing healthy eating education for midwives about cultural food preferences is recommended, and new strategies to address barriers, to ensure health services cater to the needs of diverse pregnant women should be a priority. This may include raising midwives' awareness about the scope of their role, as well as provide regular CPD education about the national and international standard healthy eating guidelines. In terms of the models of care and time allocated for antenatal appointments, maternity services need to listen to midwives' voices and adopt policies to meet their needs to enable better provision for maternity care to promote good maternal and neonatal outcomes.

\section{Funding sources}

This research is funded by a full scholarship for a PhD study provided by Cultural Affairs and Mission Sector, Ministry of Higher Education, Egyptian Government, Egypt. The funder had no role in design, data collection, analysis of the study.

\section{Ethics approval}

Ethical approval was obtained from the Human Research Ethics Committee at the UniSA (ID200150). Ethical considerations included written consent, anonymity, confidentiality, voluntary participation, and withdrawal were addressed at all stages of the research process.

\section{Conflicts of interest}

All contributing authors declare no conflicts of interest.
4. Baeyens C, Johansson C. The role of midwife during pregnancy and childbirth. Rev Med Brux. 2008;29:355-358.

5. Bahadoran P, Alizadeh S, Valiani M. Exploring the role of midwives in health care system in Iran and the world. IJNMR. 2009;14:117-122.

6. The International Confederation of Midwives 2018, Essential competencies for basic midwifery practice. https://www.internationalmidwives.org/ our-work/policy-and-practice/essential-competencies-for-midwifery-practice.html. Accessed October 7, 2019. 
7. Beldon A, Crozier S. Health promotion in pregnancy: the role of the midwife. $J R$ Soc Promot Health. 2005;125:216-220.

8. Arrish J, Yeatman H, Williamson M. Self-reported nutrition education received by Australian midwives before and after registration. J Pregnancy. 2017;5289592-5289592.

9. Arrish J, Yeatman $\mathrm{H}$, Williamson M. Midwives' role in providing nutrition advice during pregnancy: meeting the challenges? A qualitative study. Nurs Res Pract. 2017: 2017:7698510.

10. Othman SME, Steen MP, Jayasekara R, Fleet J-A. A healthy eating education program for midwives to investigate and explore their knowledge, understanding, and confidence to support pregnant women to eat healthily: protocol for a mixed-methods study. JMIR Res Protoc. 2018;7:e143-e143.

11. Othman SME, Steen M, Fleet J-A, Jayasekara R. Healthy eating in pregnancy, education for midwives: a pre-post intervention study. Eur J Midwifery. 2020;4:20.

12. Sandelowski M. Whatever happened to qualitative description? Res Nurs Health. 2000;23:334-340.

13. Bradshaw $C$, Atkinson $S$, Doody O. Employing a qualitative description approach in health care research. Glob Qual Nurs Res J. 2017;4:2333393617742282.

14. Othman S, Jayasekara R, Steen M, Fleet J. A systematic review for exploring the effectiveness of healthy eating education programmes for improving midwives' levels of knowledge and confidence in promoting healthy eating in pregnant women. Evid Based Midwifery. 2018;16:84-93.

15. Hsieh HF, Shannon SE. Three approaches to qualitative content analysis. Qual Health Res. 2005;15:1277-1288.

16. Kondracki NL, Wellman NS, Amundson DR. Content analysis: review of methods and their applications in nutrition education. J Nutr Educ Behav. 2002;34:224-230.

17. Elo $\mathrm{S}, \mathrm{Kyngäs} \mathrm{H}$. The qualitative content analysis process. J Adv Nurs. 2008;62:107-115.

18. Lincoln Y, Guba E. Naturalistic Inquiry. California: Sage Publications; 1985.

19. Miles M, Huberman M, Saldana J. Qualitative Data Analysis: A Methods Sourcebook. 3rd ed. Thousand Oaks, CA: Sage Publications; 2014.

20. Korstjens I, Moser A. Series: practical guidance to qualitative research. Part 4: Trustworthiness and publishing. Eur J Gen Pract. 2018;24:120-124.

21. Weber RP. Basic Content Analysis. California: Sage Publications; 1990.

22. Krippendorff K. Content Analysis: An Introduction to its Methodology. Newbury Park: Sage Publications; 1980.
23. Arrish J, Yeatman $\mathrm{H}$, Williamson M. Australian midwives and provision of nutrition education during pregnancy: a cross sectional survey of nutrition knowledge, attitudes, and confidence. Women Birth. 2016;29:455-464.

24. Barrowclough D, Ford F. A nutrition open-learning pack for practising midwives. Nutr Food Sci. 2001;31:6-12.

25. Arrish J, Yeatman H, Williamson M. Midwives and nutrition education during pregnancy: a literature review. Women Birth. 2014;27:2-8.

26. Yin S, Dixon L, Paterson H, Campbell N. New Zealand LMC midwives' approaches to discussing nutrition, activity and weight gain during pregnancy. N Z Coll Midwives J. 2014;50:24-29.

27. Aktaç S, Sabuncular G, Kargin D, Gunes FE. Evaluation of nutrition knowledge of pregnant women before and after nutrition education according to sociodemographic characteristics. Ecol Food Nutr. 2018;57:441-455.

28. Khoramabadi M, Dolatian M, Hajian S, et al. Effects of education based on health belief model on dietary behaviors of Iranian pregnant women. Glob $J$ Health Sci. 2015;8:230-239.

29. Elias S, Green T. Nutrition knowledge and attitudes of New Zealand registered midwives. Nutr Diet. 2007;64:290-294.

30. Baron R, Heesterbeek Q, Manniën J, Hutton EK, Brug J, Westerman MJ. Exploring health education with midwives, as perceived by pregnant women in primary care: a qualitative study in the Netherlands. Midwifery. 2017;46:37-44

31. Bondarianzadeh D, Yeatman $\mathrm{H}$, Condon-Paoloni D. A qualitative study of the Australian midwives' approaches to Listeria education as a food-related risk during pregnancy. Midwifery. 2011;27:221-228.

32. Szwajcer EM, Hiddink GJ, Koelen MA, van Woerkum CMJ. Written nutrition communication in midwifery practice: what purpose does it serve? Midwifery. 2009;25:509-517.

33. Nankumbi J, Ngabirano TD, Nalwadda G. Maternal nutrition education provided by midwives: a qualitative study in an antenatal clinic, Uganda. J Nutr Metab. 2018;2018:3987396.

34. Grimes HA, Forster DA, Newton MS. Sources of information used by women during pregnancy to meet their information needs. Midwifery. 2014;30:e26-e33.

35. Nutbeam D. The evolving concept of health literacy. Soc Sci Med. 2008;67:2072-2078.

36. Silk KJ, Sherry J, Winn B, Keesecker N, Horodynski MA, Sayir A. Increasing nutrition literacy: testing the effectiveness of print, web site, and game modalities. J Nutr Educ Behav. 2008;40:3-10. 
37. Kennard DK. Health literacy concepts in nursing education. Nurs Educ Perspect. 2016;37:118-119.

38. Cheyney M, Moreno-Black G. Nutritional counseling in midwifery and obstetric practice. Ecol Food Nutr. 2010;49:1-29.

39. Khan NN, Boyle JA, Lang AY, Harrison CL. Preconception health attitudes and behaviours of women: a qualitative investigation. Nutrients. 2019;11:1490.

40. Garnweidner LM, Sverre Pettersen K, Mosdøl A. Experiences with nutrition-related information during antenatal care of pregnant women of different ethnic backgrounds residing in the area of Oslo, Norway. Midwifery. 2013;29:e130-e137.

41. Wandel $M$, Råberg $M$, Kumar $B$, Holmboe-Ottesen $G$. Changes in food habits after migration among South Asians settled in Oslo: the effect of demographic, socio-economic and integration factors. Appetite. 2008;50:376-385.

42. Gilbert PA, Khokhar S. Changing dietary habits of ethnic groups in Europe and implications for health. Nutr Rev. 2008;66:203-215.

43. Charnley MS, McCann MT, Newson L, Rooney JS, Burden C, Abayomi JC. Midwives perceptions, knowledge and experiences of providing nutritional advice to pregnant women with obesity. Proc Nutr Soc. 2017;76:E80.

44. McCann MT, Newson L, Burden C, Rooney JS, Charnley MS, Abayomi JC. A qualitative study exploring midwives' perceptions and knowledge of maternal obesity: reflecting on their experiences of providing healthy eating and weight management advice to pregnant women. Matern Child Nutr. 2018;14:e12520.

45. Homer CSE, Biggs J, Vaughan G, Sullivan EA. Mapping maternity services in Australia: location, classification and services in Australian Health Review. Aust Health Rev. 2011;35:222-229.

46. Fenwick J, Sidebotham M, Gamble J, Creedy DK. The emotional and professional wellbeing of Australian midwives: a comparison between those providing continuity of midwifery care and those not providing continuity. Women Birth. 2018;31:38-43.

47. Yanti Y, Claramita M, Emilia O, Hakimi M. Students' understanding of "Women-Centred Care Philosophy" in midwifery care through Continuity of Care (CoC) learning model: a quasi-experimental study. BMC Nurs. 2015;14:22.

48. Homer CS. Models of maternity care: evidence for midwifery continuity of care. Med J Aust. 2016;205:370-374.

49. Sandall J, Soltani H, Gates S, ShennanA, Devane D. Midwife-led continuity models versus other models of care for childbearing women. Cochrane Database Syst Rev. 2016;4:CD004667.

50. Perriman N, Davis DL, Ferguson S. What women value in the midwifery continuity of care model: a systematic review with meta-synthesis. Midwifery. 2018; July:220-229.

51. Wilkes L, Gamble J, Creedy DK, Fenwick J. Rethinking women's access to continuity of midwife care. Women Birth. 2015;28:S47. 